\title{
Unitary Representations of the Virasoro and Super-Virasoro Algebras
}

P. Goddard ${ }^{1}$, A. Kent ${ }^{1, \star}$, and D. Olive ${ }^{2}$

1 Department of Applied Mathematics and Theoretical Physics, University of Cambridge, Silver Street, Cambridge CB3 9EW, U.K.

2 Blackett Laboratory, Imperial College, London SW7 2BZ, U.K.

\begin{abstract}
It is shown that a method previously given for constructing representations of the Virasoro algebra out of representations of affine KacMoody algebras yields the full discrete series of highest weight irreducible representations of the Virasoro algebra. The corresponding method for the super-Virasoro algebras (i.e. the Neveu-Schwarz and Ramond algebras) is described in detail and shown to yield the full discrete series of irreducible highest weight representations.
\end{abstract}

\section{Introduction}

In a recent letter [1] we described a method for constructing representations of the Virasoro algebra out of representations of affine Kac-Moody algebras. The Virasoro algebra occurs as the algebra of the conformal group in one dimension, or, in the form of two commuting copies, in two dimensions. Thus it is of importance in physical contexts where two-dimensional conformal invariance plays a crucial rôle, such as string theories or the behaviour at critical points of two-dimensional statistical systems $[2,3]$. The Virasoro algebra is defined by the commutation relations

$$
\left[L_{m}, L_{n}\right]=(m-n) L_{m+n}+\frac{c}{12} m\left(m^{2}-1\right) \delta_{m,-n}, \quad m, n \in \mathbb{Z},
$$

where $c$ is a central element, i.e. $\left[L_{n}, c\right]=0$, so that $c$ is assigned a numerical value in any irreducible representation. In this paper we shall be concerned with unitary representations of this algebra, that is representations satisfying the hermiticity conditions,

$$
L_{n}^{\dagger}=L_{-n},
$$

and, more particularly, highest weight representations, that is ones in which all the

* Present address: Enrico Fermi Institute, University of Chicago, Chicago, IL 60637, USA 
states can be generated from a highest-weight state $|h\rangle$ satisfying,

$$
\begin{gathered}
L_{n}|h\rangle=0, \quad n>0, \\
L_{0}|h\rangle=h|h\rangle .
\end{gathered}
$$

An irreducible highest-weight representation is specified by the pair of numbers $(c, h)$. It is easy to show that [3], in this case, unitarity requires

$$
c \geqq 0 \quad \text { and } \quad h \geqq 0 \text {. }
$$

Unitary representations exist for all values of $(c, h)$ with $c \geqq 1$ and $h \geqq 0$, but Friedan, Qiu, and Shenker (FQS) [3] showed that the only values of $(c, h)$ with $0 \leqq c<1$ which might correspond to unitary representations are

$$
c=1-\frac{6}{m(m+1)}, \quad m=2,3, \ldots
$$

and

$$
\begin{aligned}
& h=h_{p, q}(c) \equiv \frac{[(m+1) p-m q]^{2}-1}{4 m(m+1)}, \\
& p=1,2, \ldots, m-1 ; \quad q=1, \ldots, p .
\end{aligned}
$$

[We could extend the range of $q$ up to $m$ but this would only repeat one of the $\frac{1}{2} m(m-1)$ values above because the substitution $p \rightarrow m-p$ and $q \rightarrow m+1-q$ leaves $h_{p, q}(c)$ unchanged.]

Our construction [1], which generalises an earlier approach [4], is based on the affine Kac-Moody algebras, $\hat{g}, \hat{h}$, associated with a compact Lie group $G$ and a subgroup $H$. For each unitary representation of $\hat{g}$, and induced representation of $\hat{h}$, we obtain a representation of the Virasoro algebra. All the values of $c$ in the discrete series (1.6) can be obtained either with $G=\operatorname{Sp}(m-1)$, $H=\mathrm{Sp}(m-2) \times \mathrm{Sp}(1)$ or with $G=\mathrm{SU}(2) \times \mathrm{SU}(2)$ and $H$ being the diagonal SU (2) subgroup (and using suitable representations of $\hat{g}$ in each case). These constructions should be thought of as complementary. We shall use the second point of view here, and show that it gives all the values of $h$ given by (1.7). This demonstrates that the values of $(c, h)$ listed by FQS do indeed correspond to unitary representations.

Friedan et al. $[3,5]$ also analysed the representations of the two supersymmetric extensions of the Virasoro algebra, the Ramond [6], and Neveu and Schwarz [7] algebras, defined by (1.1) together with

$$
\begin{gathered}
{\left[L_{m}, G_{r}\right]=\left(\frac{m}{2}-r\right) G_{m+r},} \\
\left\{G_{r}, G_{s}\right\}=2 L_{r+s}+\frac{c}{3}\left(r^{2}-\frac{1}{4}\right) \delta_{r,-s},
\end{gathered}
$$

where $m \in \mathbb{Z}$ and either $r, s \in \mathbb{Z}\left[\right.$ Ramond case] or $r, s \in \mathbb{Z}+\frac{1}{2}$ [Neveu-Schwarz case]. They found that the only possible unitary highest weight representations, i.e. 
representations generated from a state $|h\rangle$ satisfying (1.3), (1.4) and

$$
G_{r}|h\rangle=0, \quad r>0,
$$

are characterised by $(c, h)$ where either $c \geqq \frac{3}{2}, h \geqq 0$ or

$$
c=\frac{3}{2}\left[1-\frac{8}{m(m+2)}\right], \quad m=2,3, \ldots
$$

and

$$
h=h_{p, q}(c) \equiv \frac{[(m+2) p-m q]^{2}-4}{8 m(m+2)}+\frac{\varepsilon}{8},
$$

where $p=1,2, \ldots, m-1$ and $q=1,2, \ldots, m+1$. Here $p-q$ even or odd corresponds to the Neveu-Schwarz and Ramond cases respectively, with $\varepsilon=0$ or $\frac{1}{2}$ correspondingly in Eq. (1.11).

A construction giving all the values of $c$ in the discrete series (1.10) was sketched in [1]. It is described in more detail in Sect. 3. In Sect. 4 we demonstrate that it too produces all the corresponding values of $h$ in Eq. (1.11), completing the classification of unitary highest weight representations of the Neveu-Schwarz and Ramond algebras.

\section{Unitary Representation of the Virasoro Algebra}

The methods of constructing representations of Virasoro algebras given in [1, 4] start from the Virasoro algebras one can construct from affine Kac-Moody algebras. If $g$ is a simple Lie algebra,

$$
\left[T^{a}, T^{b}\right]=i f^{a b c} T^{c},
$$

written in a basis in which the structure constants $f^{a b c}$ are totally antisymmetric, the associated affine Kac-Moody algebra $\hat{g}$ takes the form

$$
\left[T_{m}^{a}, T_{n}^{b}\right]=i f^{a b c} T_{m+n}^{c}+k m \delta^{a b} \delta_{m,-n},
$$

$m, n \in \mathbb{Z}$, where $k$ is a central element (and so is assigned a numerical value in any irreducible representation). We shall be concerned with unitary highest weight representations of $\hat{g}$ also, that is representations in a positive definite Hilbert space satisfying

$$
T_{n}^{a \dagger}=T_{-n}^{a},
$$

and with the representation space generated from vacuum states $\Psi$ satisfying

$$
T_{n}^{a} \Psi=0, \quad n>0 .
$$

The central element $k$ is quantised in multiples of $\frac{1}{2} \psi^{2}$, where $\psi$ is a long root of $g$. The integer $2 k / \psi^{2}$ is called the level.

From $\hat{g}$ can be constructed [8] a Virasoro algebra $L_{n}^{g}$ defined by

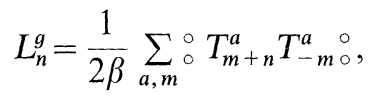


where the normal ordering operation is defined by

$$
{ }_{\circ}^{\circ} T_{n}^{a} T_{-n}^{a} \stackrel{\circ}{\circ}=T_{-n}^{a} T_{n}^{a} \quad \text { if } n \geqq 0,
$$

and

$$
\beta=k+\frac{1}{2} c_{\psi}^{g} .
$$

Here $c_{\psi}^{g}$ denotes the quadratic Casimir operator for the adjoint representation of $g$,

$$
f^{a b c} f^{a b d}=c_{\psi}^{g} \delta^{c d}
$$

The $L_{n}^{g}$ satisfy (1.1) with $c$ taking the value

$$
c^{g}=k \operatorname{dim} g / \beta=\frac{2 k \operatorname{dim} g}{c_{\psi}^{g}+2 k} .
$$

Clearly if we start with a unitary representation of $\hat{g}$, this provides a unitary representation of the Virasoro algebra.

If $g$ is semisimple there is a central element $k_{i}$ in $\hat{g}$ for each simple factor $g_{i}$, $1 \leqq i \leqq N$, of $g$. Then

$$
L_{n}^{g}=\sum_{i=1}^{N} L_{n}^{g_{2}}
$$

is a Virasoro algebra with central element

$$
c^{g}=\sum_{i=1}^{N} c^{g_{i}}=\sum_{i=1}^{N} \frac{2 k_{i} \operatorname{dim} g_{i}}{c_{\psi}^{g_{i}}+2 k_{i}} .
$$

The main idea in [1] was to consider not only an algebra $g$ but also a subalgebra $h \subset g$. We label the basis for $g$ so that the first $\operatorname{dim} h$ generators form a basis for $h$. In this situation we have two Virasoro algebras $L_{n}^{g}, L_{n}^{h}$ and we can consider their difference

$$
K_{n}=L_{n}^{g}-L_{n}^{h}
$$

which satisfies the Virasoro algebra with central element

$$
c=c^{g}-c^{h}
$$

and commutes with $\hat{h}$,

$$
\left[K_{m}, T_{n}^{a}\right]=0, \quad 1 \leqq a \leqq \operatorname{dim} h,
$$

$m, n \in \mathbb{Z}$. It is not difficult to see that a unitary highest weight representation of $\hat{g}$ provides a unitary highest weight representation of $K_{n}$ and so, necessarily,

$$
c^{g} \geqq c^{h} .
$$

Let us now consider what are the possibilities for highest weight irreducible unitary representations of $\hat{g}$. (For reviews see [9-11].) In such a representation the vacuum states $\Psi$ satisfying (2.4) form a finite-dimensional irreducible representation of $g \cong\left\{T_{0}^{a}\right\}$ whose highest weight $\lambda$ must satisfy

$$
|\alpha \cdot \lambda| \leqq k
$$


for all roots $\alpha$ of $g$, which is assumed simple for the moment. This taken, together with the condition $2 k / \psi^{2} \in \mathbb{Z}$, is a necessary and sufficient condition for the existence of an irreducible unitary representation of $\hat{g}$. This representation can be labelled by $\left(2 k / \psi^{2}, \lambda\right)$. If $g$ is not simple, the irreducible representations of $\hat{g}$ can be constructed from those of $\hat{g}_{i}$, where the $g_{i}$ are the factors of $g$.

In [1] it was remarked that the values of $c$ in the discrete series (1.6) could be obtained by taking $g=\mathrm{su}(2) \oplus \mathrm{su}(2)$ and $h$ to be the diagonal su(2) subalgebra. The $\mathrm{su}(2)$ (affine untwisted) Kac-Moody algebra can be written in the form

$$
\left[T_{m}^{a}, T_{n}^{b}\right]=i \varepsilon_{a b c} T_{m+n}^{c}+\frac{N}{2} m \delta^{a b} \delta_{m,-n}
$$

and then $\psi^{2}=1$. It follows that $N \in \mathbb{Z}$ for a unitary highest weight representation and is the level of that representation. Such representations are labelled by $(N, l)$ where $l$ is the highest helicity (i.e. the largest eigenvalue of $T=T_{0}^{3}$ ) for a vacuum state $\Psi$. We shall call this the level $N$, spin $l$ representation; condition (2.16) implies that $0 \leqq 2 l \leqq N$. A level $N$ representation of sû( 2$)$ gives rise to a Virasoro algebra with central element

$$
c=\frac{3 N}{N+2} .
$$

If we construct a representation of $\hat{g}$ for $g=s u(2) \oplus s u(2)$ by taking a level $N$ representation of the first factor and a level 1 representation of the second factor, we obtain a level $N+1$ representation of the diagonal $h=\mathrm{su}(2)$. Thus $K_{n}$ given by Eq. (2.12) has a value of $c$, given by (2.13),

$$
c=1-\frac{6}{(N+2)(N+3)} \text {. }
$$

Taking $N=0,1, \ldots$ gives the sequence of Eq. (1.6).

Our aim now is to show that from such representations of $K_{n}$ we can obtain all the values of $h$ given by Eq. (1.7). To do this we decompose the level $(N, 1)$ representation of $\hat{g}$ with respect to $\hat{h} \times V$, where $V$ denotes the Virasoro algebra $\left\{K_{n}\right\}$. We shall show that the representation obtained by taking the $(N, l)$ representation of the first su(2) factor and the $(1, \varepsilon), \varepsilon=0$ or $\frac{1}{2}$, representation of the second $\mathrm{su}(2)$ factor decomposes into the direct sum of representations $\left(N^{\prime}, l^{\prime}\right) \times(c, h)$ of $\hat{h} \times V$,

$$
\bigoplus_{q}\left(N+1, \frac{1}{2}[q-1]\right) \times\left(c, h_{p, q}(c)\right),
$$

where $c$ is given by Eq. (2.19); $p=2 l+1$, so that $1 \leqq p \leqq N+1$, and the sum is taken over $q$ such that $p-q$ is even or odd, depending on whether $\varepsilon=0$ or $\frac{1}{2}$, and $1 \leqq q$ $\leqq N+2$. This gives all the values required. Note that this implies that the representation $(N, l) \times(1, \varepsilon)$ of $\hat{g}$ is finitely and simply reducible in terms of $\hat{h} \times V$.

To establish (2.20) we introduce characters for the algebras we are studying, though it turns out that we shall only need to make a very limited appeal to the theory of characters. Essentially all we need are the Kac-Moody formula [9] and a formula for the characters of the Virasoro algebra [12], but we shall explain these as we develop the argument. 
We define the character $\chi_{N, l}$ for the level $N$ spin $l$ representation of the $\operatorname{su}(2)$ Kac-Moody algebra by

$$
\chi_{N, l}(z, \theta)=\operatorname{tr}\left(z^{L_{0}} e^{i \theta T_{0}^{3}}\right)
$$

In the level 1 case, where $\varepsilon=0, \frac{1}{2}$, there is a rather explicit formula for $\chi_{1, \varepsilon}$ which can be deduced from the vertex operator construction of these representations [13, 14]. An account of this and its relation to string theory is given in [15]. Because these representations can be constructed irreducibly in a Fock space defined by the annihilation and creation operators $\alpha_{m}$ acting on momentum eigenstates $|\gamma\rangle$, $\gamma / \sqrt{2} \in \mathbb{Z}+\varepsilon$, where

$$
\begin{gathered}
{\left[\alpha_{m}, \alpha_{n}\right]=m \delta_{m,-n}, \quad m, n \in \mathbb{Z},} \\
\alpha_{m}^{\dagger}=\alpha_{-m}, \quad \alpha_{m}|\gamma\rangle=0, \quad m>0, \\
p|\gamma\rangle=\gamma|\gamma\rangle, \quad \alpha_{0} \equiv p,
\end{gathered}
$$

with

$$
L_{0}=\frac{1}{2} p^{2}+\sum_{n>0} \alpha_{-n} \alpha_{n}, \quad T=p / \sqrt{2}
$$

we have

$$
\chi_{1, \varepsilon}(z, \theta)=\sum_{m \in \mathbb{Z}+\varepsilon} z^{m^{2}} e^{i m \theta} \prod_{n=1}^{\infty}\left(1-z^{n}\right)^{-1} .
$$

To obtain the characters for other representations of the su(2) Kac-Moody algebra we need to resort to the Kac-Weyl formula, of which some explanation is given in Appendix A. In the $\mathrm{su}(2)$ case this gives the expression

$$
\chi_{N, l}(z, \theta)=\Delta_{N, l}(z, \theta) \prod_{n=1}^{\infty}\left(1-z^{n}\right)^{-1}\left(1-z^{n} e^{i \theta}\right)^{-1}\left(1-z^{n-1} e^{-i \theta}\right)^{-1},
$$

where the numerator

$$
\Delta_{N, l}(z, \theta)=z^{l(l+1) / \lambda} \sum_{n \in \mathbb{Z}} z^{\lambda n^{2}+(2 l+1) n}\left\{e^{i(l+\lambda n) \theta}-e^{-i(l+1+\lambda n) \theta}\right\}
$$

with

$$
\lambda=N+2 .
$$

We can regard Eq. (2.25) as defining a function for all values of $N$ and $l$. With this extension, it is straightforward to establish directly the symmetry properties

$$
\begin{gathered}
\Delta_{N, l+\lambda}(z, \theta)=\Delta_{N, \lambda}(z, \theta), \\
\Delta_{N,-l-1}(z, \theta)=-\Delta_{N, l}(z, \theta),
\end{gathered}
$$

which follow from the symmetry of characters under the action of the Weyl group of $\hat{g}$.

The other character formulae we need are those for representations of the Virasoro algebra. The character of the representation $(c, h)$ is defined by the trace

$$
\chi_{c, h}^{V}(z)=\operatorname{tr}\left(z^{L_{0}}\right) \text {. }
$$


For the discrete series of Eq. (1.6), (1.7) these have been given by RochaCaridi [12],

$$
\chi_{c, h}^{V}(z)=\Delta_{p, q}^{m}(z) \prod_{n=1}^{\infty}\left(1-z^{n}\right)^{-1},
$$

where

$$
\Delta_{p, q}^{m}(z)=\sum_{n \in \mathbb{Z}}\left\{z^{\alpha_{p}^{m}, q^{(n)}}-z^{\beta_{p}^{m}, q^{(n)}}\right\}
$$

with

$$
\begin{aligned}
& \alpha_{p, q}^{m}(n)=\frac{[2 m(n+1) n-q m+p(m+1)]^{2}-1}{4 m(m+1)}, \\
& \beta_{p, q}^{m}(n)=\frac{[2 m(m+1) n+q m+p(m+1)]^{2}-1}{4 m(m+1)} .
\end{aligned}
$$

These expressions follow from the general results of Feigin and Fuchs [16] on the structure of Virasoro algebra representations. For a proof and discussion of these results see [17].

Let us consider what (2.20) is equivalent to in terms of characters. Because the $T$ for $\hat{h}$ is the sum of the T's for the two su(2) factors in $\hat{g}$, and because

$$
L_{0}^{1}+L_{0}^{2}=L_{0}^{h}+K_{0},
$$

where $L_{n}^{i}, i=1,2$, denotes the Virasoro algebra for the two su(2) factors of $\hat{g}$, the decomposition (2.20) implies

$$
\chi_{N, l}(z, \theta) \chi_{1, \varepsilon}(z, \theta)=\sum_{q} \chi_{N+1, \frac{1}{2}(q-1)}(z, \theta) \chi_{c, h}^{V}(z)
$$

with $c, h, p, q$ specified as in Eq. (2.20). On the other hand, if Eq. (2.34) holds we can use it to decompose the given representation of $\hat{g}$ into irreducible representations of $\hat{h} \times V$, because from it we can successively isolate highest weight states for the algebra $\hat{h} \times V$, that is, states $\Psi_{h}^{j m}$ satisfying

$$
\begin{gathered}
T_{n}^{i} \Psi_{h}^{j m}=0, \quad K_{n} \Psi_{h}^{j m}=0, \quad n>0, \\
K_{0} \Psi_{h}^{j m}=h \Psi_{h}^{j m}, \\
T_{0}^{3} \Psi_{h}^{j m}=m \Psi_{h}^{j m}, \quad\left(T_{0}^{i}\right)^{2} \Psi_{k}^{j m}=j(j+1) \Psi_{h}^{j m} .
\end{gathered}
$$

To do this we look for the lowest powers of $z$ on the right-hand side of (2.34). These will be a sum of terms of the form

$$
z^{h+\frac{1}{2} L(L+1)} \frac{\sin \left(L+\frac{1}{2}\right) \theta}{\sin \frac{1}{2} \theta},
$$

where $L=\frac{1}{2}(q-1)$. Such a term indicates the presence of highest weight states $\Psi_{h}^{j m}$ with $j=L$ and a representation $(N+1, L) \times(c, h)$ of $\hat{h} \times V$. Removing the states of this representation corresponds to subtracting the term

$$
\chi_{N+1, \frac{1}{2}(q-1)}(z, \theta) \chi_{c, h}^{V}(z)
$$

from (2.34). Proceeding inductively in this way one establishes (2.20). 
It remains to prove (2.34) using the expressions (2.23)-(2.25) and (2.30)-(2.32). Because the denominators match, it is equivalent to

$$
\left\{\sum_{m \in \mathbb{Z}+\varepsilon} z^{m^{2}} e^{i m \theta}\right\} \Delta_{N, l}(z, \theta)=\sum_{q} \Delta_{N+1, \frac{1}{2}(q-1)}(z, \theta) \Delta_{p, q}^{N+2}(z) .
$$

Our strategy in proving this is to rewrite the left-hand side of (2.38) as a sum over functions $\Delta_{N+1, j}(z, \theta)$, multiplied by functions of $z$ only, and then use the symmetry properties $(2.27),(2.28)$ to bring $j$ into the appropriate range that $\frac{1}{2}(q-1)$ goes over. Using (2.25), this left-hand side equals

$$
z^{l(l+1) / \lambda} \sum_{m \in \mathbb{Z}+\varepsilon} \sum_{n \in \mathbb{Z}} z^{\lambda n^{2}+(2 l+1) n+m^{2}}\left\{e^{i(l+\lambda n+m) \theta}-e^{-i(l+1+\lambda n+m) \theta}\right\} .
$$

Now put $m^{\prime}=l+m-n$ so that $m^{\prime} \in \mathbb{Z}+\varepsilon^{\prime}$, where $\varepsilon^{\prime}=0$ or $\frac{1}{2}$ as $l+\varepsilon \in \mathbb{Z}$ or $\mathbb{Z}+\frac{1}{2}$. Then

$$
l+m+\lambda n=m^{\prime}+(\lambda+1) n
$$

and

$$
\lambda n^{2}+(2 l+1) n+m^{2}=(\lambda+1) n^{2}+\left(2 m^{\prime}+1\right) n+\left(m^{\prime}-l\right)^{2} .
$$

Thus

$$
\left\{\sum_{m \in \mathbb{Z}+\varepsilon} z^{m^{2}} e^{i m \theta}\right\} \Delta_{N, l}(z, \theta)=\sum_{m^{\prime} \in \mathbb{Z}+\varepsilon^{\prime}} z^{A} \Delta_{N+1, m^{\prime}}(z, \theta),
$$

where

$$
A=l(l+1) / \lambda-m^{\prime}\left(m^{\prime}+1\right) /(\lambda+1)+\left(m^{\prime}-l\right)^{2} .
$$

Now put

$$
m^{\prime}=-(\lambda+1) M+l^{\prime}
$$

where $M \in \mathbb{Z}$ and $0 \leqq l^{\prime} \leqq \lambda$. Also put

$$
p=2 l+1, \quad q=2 l^{\prime}+1
$$

so that $p, q \in \mathbb{Z}, 1 \leqq q \leqq 2 \lambda+1,1 \leqq p \leqq \lambda-1$ and $p-q$ is even or odd as $\varepsilon=0$ or $\frac{1}{2}$. Then

$$
A=\alpha_{p, q}^{\lambda}(M)
$$

as defined by Eq. (2.32a). Using the periodicity property (2.27), the character becomes

$$
\sum_{1 \leqq q \leqq 2 \lambda+1} \Delta_{N+1, l^{\prime}}(z, \theta)\left\{\sum_{M} z^{\alpha_{p}^{\lambda}, q(M)}\right\} .
$$

If we use the reflection property (2.28), substituting $(\lambda+1)$ for $\lambda$ here, together with the relation

$$
\alpha_{p, 2 \lambda+2-q}^{\lambda}(M)=\beta_{p, q}^{\lambda}(M-1),
$$

we obtain the right-hand side of Eq. (2.30), thus completing our proof. 


\section{Construction of the Super-Virasoro Algebras}

In this section we describe in more detail the construction, sketched in a special case in [1], of operators satisfying the super-Virasoro algebra of Eqs. (1.1) and (1.8). The framework within which we work is again that of the Kac-Moody algebras $\hat{g}, \hat{h}$ associated with the Lie algebras $g \supset h$. We shall take $g$ to have the form $g=h_{T} \oplus h_{v}$, where $h_{T}$ and $h_{v}$ are isomorphic, and $h$ is their diagonal subalgebra, i.e. the form used in the last section with $h=\mathrm{su}(2)$ to obtain all the discrete series representations of the Virasoro algebra.

The representations of $\hat{h}_{v}$ that we shall use will be defined in fermionic Fock spaces [18] built up from fermionic fields in the adjoint representation of $h$; for $\hat{h}_{T}$ we can use any highest weight unitary representation. In this situation, the Virasoro algebra

$$
K_{n}=L_{n}^{g}-L_{n}^{h}
$$

can be extended by fermionic generators $G_{r}$, satisfying

$$
\left[K_{n}, G_{r}\right]=\left(\frac{n}{2}-r\right) G_{n+r}
$$

and

$$
\left\{G_{r}, G_{s}\right\}=2 K_{r+s}+\frac{c}{3}\left(r^{2}-\frac{1}{4}\right) \delta_{r,-s},
$$

where either $r, s \in \mathbb{Z}$ or $r, s \in \mathbb{Z}+\frac{1}{2}$, consistently, thus giving the Ramond and Neveu-Schwarz algebras respectively.

In the context where $g=h_{T} \oplus h_{v}$, with both $\hat{h}_{T}$ and $\hat{h}_{v}$ being represented by fermion fields (in the latter case in the adjoint representations) there are a number of super-Virasoro algebras that can be constructed. Of these, the supersymmetric extension of $K_{n}$ is the least expected. We shall discuss these constructions and their significance elsewhere [19]. Here our objective is to show that for $h=s u(2)$ the $K_{n}, G_{r}$ algebra produces the full discrete series of representations of the Ramond and Neveu-Schwarz algebras.

To construct the representation of $h_{v}$ that we need we introduce $\operatorname{dim} h$ fermion fields

$$
H(z)=\sum_{r} b_{r}^{a} z^{-r}, \quad 1 \leqq a \leqq \operatorname{dim} h,
$$

where the sum is over either $r \in \mathbb{Z}$ (Ramond [R] case) or $r \in \mathbb{Z}+\frac{1}{2}$ (Neveu-Schwarz [NS] case), and the fermi oscillators satisfy

$$
\begin{gathered}
\left\{b_{r}^{a}, b_{s}^{b}\right\}=\delta^{a b} \delta_{r,-s}, \\
b_{r}^{a}|0\rangle=0, \quad r>0, \quad b_{r}^{a \dagger}=b_{-r}^{a} .
\end{gathered}
$$

In the NS case the vacuum $|0\rangle$ is non-degenerate, whilst in the $R$ case the vacua form a representation of the Dirac algebra $b_{0}^{a}, 1 \leqq a \leqq \operatorname{dim} h$. We define fermion 
normal ordering by

$$
\begin{aligned}
: b_{r}^{a} b_{s}^{b}: & =b_{r}^{a} b_{s}^{b}, & & r<0, \\
& =-b_{s}^{b} b_{r}^{a}, & & r>0, \\
& =\frac{1}{2}\left[b_{r}^{a}, b_{s}^{b}\right], & & r=0 .
\end{aligned}
$$

The representation $V_{m}^{a}$ of $\hat{h}_{v}$ is defined by

$$
V^{a}(z) \equiv \sum V_{n}^{a} z^{-n}=-\frac{i}{2} f^{a b c}: H^{b}(z) H^{c}(z):
$$

From this it follows that [4]

$$
\left[V_{m}^{a}, V_{m}^{b}\right]=i f^{a b c} V_{m+n}^{c}+v m \delta^{a b} \delta_{m,-n},
$$

where $v=\frac{1}{2} c_{\psi}^{h}$. Thus we have a (reducible) representation of $\hat{h}_{v} \cong \hat{h}$ of level $c_{\psi}^{h} / \psi^{2}$.

For $h_{T}$ we take a highest weight irreducible representation labelled by $\left(2 k / \psi^{2}, \lambda\right)$

$$
T^{a}(z) \equiv \sum T_{n}^{a} z^{-n}
$$

Then the Virasoro algebra $K_{n}$ is defined by Eq. (3.1) where

$$
L^{g}(z)=L^{h_{T}}(z)+L^{h_{v}}(z)=\frac{1}{2(k+v)} \stackrel{\circ}{\circ} T^{a}(z) T^{a}(z)_{\circ}^{\circ}+\frac{1}{4 v} \circ V^{a}(z) V^{a}(z) \circ,
$$

and

$$
L^{h}(z)=\frac{1}{2(k+2 v)} \stackrel{\circ}{\circ}\left[T^{a}(z)+V^{a}(z)\right]\left[T^{a}(z)+V^{a}(z)\right]_{\circ}^{\circ} .
$$

It has central element

$$
c=C^{h_{T}}+C^{h_{v}}-C^{h}=\frac{k(k+3 v) \operatorname{dim} h}{2(k+v)(k+2 v)} .
$$

From Eq. (2.14) it follows that

$$
\left[K_{m}, T_{n}^{a}+V_{n}^{a}\right]=0, \quad 1 \leqq a \leqq \operatorname{dim} h,
$$

and it is natural to seek super-Virasoro operators $G_{r}$ which also commute with $\hat{h}$. Candidate building-blocks for $G(z)$ are $T^{a}(z) H^{a}(z)$ and

$$
\frac{1}{3}: V^{a}(z) H^{a}(z):=-\frac{i}{6}: f^{a b c} H^{a}(z) H^{b}(z) H^{c}(z): .
$$

Straightforward calculation shows that

$$
G(z) \equiv \sum_{r} G_{r} z^{-n}=[v(k+v)(k+2 v)]^{-1 / 2}\left[k T^{a}(z) H^{a}(z)-\frac{v}{3}: V^{a}(z) H^{a}(z):\right](3
$$

provides a supersymmetric extension of $K_{n}$ satisfying (3.2), (3.3) and commuting with $\hat{h}$. 
The construction we have given in this section can be applied to any Lie algebra $h$, using in its adjoint representation and any representation of $\hat{h} \cong \hat{h}_{T}$. One can equally regard this as an irreducible representation of the super-affine KacMoody algebra based on $h[20]$. However, we have found no underlying reason for the existence of this particular super-Virasoro algebra. For further discussion see [19].)

\section{Unitary Representations of the Super-Virasoro Algebras}

To find the whole of the discrete series of unitary representations we use the construction of the last section in the particular case in which $h \cong \operatorname{su}(2)$. For the su(2) Kac-Moody algebra $\hat{h}_{T}$ we use the level $N$, spin $l$ irreducible representation, whilst for $\hat{h}_{v}$ we must use the adjoint fermion representation described in Sect. 3. This is a level 2 representation which in the NS case decomposes into spin 0 and spin 1 irreducible components, with highest weight states $|0\rangle$ and $b_{-1 / 2}^{i}|0\rangle$, whilst in the $R$ case the vacuum can be taken to be 2-fold degenerate, and then the representation will be an irreducible level 2, spin $\frac{1}{2}$ representation. From Sect. 3, it follows that, acting on the resulting representation space for $\hat{g}$, we have an su(2) Kac-Moody algebra $\hat{h}$ and the super-Virasoro algebras $s=\left\{K_{n}, G_{r}\right\}$. Thus we can parallel the discussion of Sect. 2 by decomposing the resulting representations of $\hat{g}$ with respect to $\hat{h} \times s$. The central element in $s$ obtained in this way is [1]

$$
c=\frac{3}{2}\left(1-\frac{8}{(N+2)(N+4)}\right),
$$

providing the whole of the discrete series of values of $c$. We need to show that we also obtain all the corresponding values of $h_{p, q}$ listed in Eq. (1.11).

The characters of the adjoint fermion representations, as defined by (2.20), can be written down directly using their Fock space construction,

$$
\chi_{2}^{\mathrm{NS}}(z, \theta)=\prod_{n=1}^{\infty}\left(1+z^{n-1 / 2}\right)\left(1+z^{n-1 / 2} e^{i \theta}\right)\left(1+z^{n-1 / 2} e^{-i \theta}\right),
$$

and

$$
\chi_{2}^{R}(z, \theta)=z^{3 / 16}\left(e^{i \theta / 2}+e^{-i \theta / 2}\right) \prod_{n=1}^{\infty}\left(1+z^{n}\right)\left(1+z^{n} e^{i \theta}\right)\left(1+z^{n} e^{-i \theta}\right) .
$$

Here we have used the fact that for this representation [4], $L^{g}(z)$ is the same as

$$
L(z)=\frac{i}{2}: \frac{d H^{a}}{d z} H^{a}(z):+\frac{3 \varepsilon}{8} .
$$

Using the Jacobi triple product formula

$$
\prod_{n=1}^{\infty}\left(1-x^{n}\right)\left(1+x^{n-1} w\right)\left(1+x^{n} / w\right)=\sum_{n \in \mathbb{Z}} x^{1 / 2 n(n-1)} w^{n},
$$

we can rewrite these characters in the forms

$$
\chi_{2}^{\mathrm{NS}}(z, \theta)=\sum_{m \in \mathbb{Z}} z^{1 / 2 m^{2}} e^{i m \theta} \prod_{n=1}^{\infty}\left(\frac{1+z^{n-1 / 2}}{1-z^{n}}\right)
$$


and

$$
\chi_{2}^{R}(z, \theta)=z^{1 / 16} \sum_{m \in \mathbb{Z}+\frac{1}{2}} z^{1 / 2 m^{2}} e^{i m \theta} \prod_{n=1}^{\infty}\left(\frac{1+z^{n}}{1-z^{n}}\right) .
$$

If we denote these representations of $\operatorname{su}(2)$ by $(2, \mathrm{NS})$ and $(2, R)$, as we have just remarked,

$$
\begin{gathered}
(2, \mathrm{NS})=(2,0) \oplus(2,1), \\
(2, R)=\left(2, \frac{1}{2}\right) .
\end{gathered}
$$

In order to repeat the argument of Sect. 2, we also need to know the characters of the discrete series of super-Virasoro algebra representations. For the representations $\left(c, h_{p, q}\right)$ of Eqs. $(1.10),(1.11)$ these are given by [17]

$$
\chi_{c, h}^{\mathrm{NS}}(z)=\Gamma_{p, q}^{m}(z) \prod_{n=1}^{\infty}\left(\frac{1+z^{n-1 / 2}}{1-z^{n}}\right)
$$

and

$$
\chi_{c, h}^{R}(z)=\Gamma_{p, q}^{m}(z) z^{1 / 16} \prod_{n=1}^{\infty}\left(\frac{1+z^{n}}{1-z^{n}}\right),
$$

where $1 \leqq p \leqq m-1,1 \leqq q \leqq m+1$ and $p-q$ is even in the NS case and odd in the $R$ case. The functions $\Gamma_{p, q}^{m}(z)$ are defined by an equation similar to (2.31),

$$
\Gamma_{p, q}^{m}(z)=\sum_{m \in \mathbb{Z}}\left\{Z^{\gamma_{P, q}^{m}(n)}-Z^{\delta_{P, q}^{m}(n)}\right\},
$$

where

$$
\gamma_{p, q}^{m}(n)=\frac{[2 m(m+1) n-p(m+2)+m q]^{2}-4}{8 m(m+2)}
$$

and

$$
\delta_{p, q}^{m}(n)=\frac{[2 m(m+1) n+p(m+2)+m q]^{2}-4}{8 m(m+2)} .
$$

We can now establish the identity

$$
\left\{\sum_{m \in \mathbb{Z}+\varepsilon} z^{m^{2}} e^{i m \theta}\right\} \Delta_{N, \frac{1}{2}(p-1)}(z, \theta)=\sum_{q} \Delta_{N+2, \frac{1}{2}(q-1)}(z, \theta) \Gamma_{p, q}^{N+2}(z),
$$

where the sum over $q$ is over values lying in the range $1 \leqq q \leqq N+3$ and with $p-q \in 2 \mathbb{Z}+2 \varepsilon$, using exactly the same sort of manipulation that were used in Sect. 2 to prove Eq. (2.38). Thus it follows that

$$
\chi_{N, \frac{1}{2}(p-1)}(z, \theta) \chi_{2}^{F}(z, \theta)=\sum_{q} \chi_{N+2, \frac{1}{2}(q-1)}(z, \theta) \chi_{c, h}^{F}(z),
$$

where $F$ stands either for NS or $R, h=h_{p, q}(c), c$ is as in Eq. (4.1) and the range of $q$ is as in Eq. (4.13) with $\varepsilon=0$ in the NS case and $\varepsilon=\frac{1}{2}$ in the $R$ case. By the same arguments used in Sect. 2, Eq. (4.14) implies the decompositions

$$
\left(N, \frac{1}{2}[p-1]\right) \times(F, 2)=\bigoplus_{q}\left(N+2, \frac{1}{2}[q-1]\right) \times\left(c, h_{p, q}(c)\right)_{F},
$$


where $(c, h)_{F}$ denotes the appropriate representation of the NS or $R$ superVirasoro algebras, as $F=\mathrm{NS}$ or $R$, and the rest of the notation is as in Eq. (4.14). This provides the whole of the discrete series of unitary representations of the super-Virasoro algebras and establishes the unitarity.

\section{Comments}

In this paper we have established the existence of the full discrete series of representations of the Virasoro and super-Virasoro algebras starting from irreducible representations of the (affine untwisted) su(2) Kac-Moody algebra. In [1] we based our construction on the representations of this algebra which could be constructed using NS or $R$ fermion fields transforming under spin $\frac{1}{2}$ or spin 1 representations of su(2). We did not show that all the values of $h$ given by Eqs. (1.7) and (1.11) could be found in this context (thus leaving the existence of all the discrete series representations still in doubt) but in fact they can [17].

Another approach to the construction of Virasoro algebra, but not superVirasoro algebra construction, described in [1], was to use the inclusion

$$
\operatorname{Sp}(n-1) \times \operatorname{Sp}(1) \subset \operatorname{Sp}(n),
$$

and this can also be used to obtain all the representations for the Virasoro algebra. This approach has interesting applications and it will be described in [17]. Altschuler [21] has verified that within this latter construction the Ramond vacuum yields the eigenvalues $h_{p, p}$ and $h_{p, p-1}(1 \leqq p \leqq m-1)$.

\section{Appendix. The Weyl-Kac Character Formula}

The aim of this appendix is to write down and motivate the Weyl-Kac formula for the character of a highest weight unitary irreducible representation of the affine Kac-Moody algebra, indicating that it is the natural generalisation of the Weyl formula for the character of a unitary irreducible representation of the finite dimensional Lie algebra $g$. We will show how, when $g$ is chosen to be su(2), it yields the character formula (2.24) used in the text.

Let $\lambda$ be the highest weight of a unitary irreducible representation of $g$ (so that it is integral and dominant). Then the character of this representation is given by

$$
\chi^{\lambda}(\theta)=\operatorname{tr}\left(e^{i \theta \cdot T}\right)=\sum_{\sigma \in W(g)} \varepsilon(\sigma) e^{i \sigma(\lambda+\varrho) \cdot \theta} e^{-i \varrho \cdot \theta} \prod_{\alpha>0}\left(1-e^{-i \alpha \cdot \theta}\right)^{-1},
$$

where $\varepsilon(\sigma)=\operatorname{det} \sigma, W(g)$ denotes the Weyl group of $g$, and $\varrho$ denotes half the sum of the positive roots of $g$. As $W(\mathrm{su}(2))$ has just two elements, $\pm 1,(\mathrm{~A} .1)$ reduces to the familiar formula

$$
\chi^{L}(\theta)=\frac{\sin \left(L+\frac{1}{2}\right) \theta}{\sin \frac{1}{2} \theta}
$$

occurring in Eq. (2.36).

Both sides of (A.1) can be assigned a meaning for the Kac-Moody algebra $\hat{g}$, since $\hat{g}$ still has finite rank, a system of positive roots and a Weyl group $W(\hat{g})$ generated by reflections in real roots, which is in fact isomorphic to the semidirect product of $W(g)$ with the co-root lattice of $g, \Lambda_{r}^{v}$. For further explanation of these 
results and the notation we are about to adopt we refer to a review by one of us [11].

The Cartan subalgebra of $\hat{g}$, extended by derivation $d=-L_{0}$, is taken to have basis $\left(T^{i}, 1 \leqq i \leqq \operatorname{rank} g, k, d\right)$. In an irreducible unitary highest weight representation, $k$ takes a fixed value, $\frac{1}{2} x \psi^{2}$, where $x$ is the level, while $L_{0}$ exceeds its smallest value, $-\delta$, by positive integers. Thus it is meaningful to adopt the following definition of the character.

$$
\chi_{x, l}(z, \theta) \equiv \operatorname{tr}\left(z^{L_{0}} e^{i \theta \cdot T}\right)
$$

with convergence expected for $|z|<1$. Formally, we can rewrite this as follows in order to compare with (A.1),

$$
\chi_{x, l}(z, \theta)=\operatorname{tr}\left\{e^{i(\theta \cdot T+i(\ln z) d+o \cdot k)}\right\} .
$$

Now the Kac-Moody analogue in (A.4) of $\lambda+\varrho$ in (A.1) is

$$
y=\left(\lambda+\varrho, \frac{1}{2} \psi^{2}(x+\tilde{h}), \delta\right),
$$

where $\tilde{h}$, the "level" of $\varrho$, is the dual Coxeter of $g$, and $\delta$ is yet to be determined by comparing the leading $z$ behaviour of the character. The action of a Weyl reflection $S_{a}$ in a root of $\hat{g}$ is, according to Sect. 8 of [11],

$$
S_{a}(y)=\left(\sigma_{a}(\lambda+\varrho+t), \frac{1}{2} \psi^{2}(x+\tilde{h}), \delta+\left[(\lambda+\varrho)^{2}-(\lambda+\varrho+t)^{2}\right] /(x+\tilde{h}) \psi^{2}\right),
$$

where

$$
t=n \alpha(x+\tilde{h}) \psi^{2} / \alpha^{2},
$$

and so is proportional to an element of the co-root lattice of $g$.

Thus we expect the Kac-Moody analogue of (A.1) to read

$$
\chi_{x, \lambda}(z, \theta)=\Delta_{x, \lambda}(z, \theta) / \Delta_{0}(z, \theta),
$$

where

$$
\Delta_{x, \lambda}(z, \theta)=\sum_{t \in \Lambda_{r}^{v}} z^{-\delta+\left[(\lambda+\varrho+t)^{2}-(\lambda+\varrho)^{2}\right] /(x+\tilde{h}) \psi^{2}} \sum_{\sigma \in W(g)} \varepsilon(\sigma) e^{i \sigma(\lambda+\varrho+t) \cdot \theta}
$$

and

$$
\Delta_{0}(z, \theta)=e^{i \varrho \cdot \theta} \prod_{n=1}^{\infty}\left\{\left(1-z^{n}\right)^{r} \prod_{\alpha>0}\left(1-z^{n-1} e^{-i \alpha \cdot \theta}\right)\left(1-z^{n} e^{i \alpha \theta}\right)\right\}
$$

using the structure of the root system and Weyl group of $\hat{g}$. It follows that the leading term in $z$ occurring in $\chi_{x, \lambda}(z, \theta)$ is $z^{-\delta} \chi_{\lambda}(\theta)$. Since the states of lowest $L_{0}$ value form an irreducible representation of $g$ with highest weight $\lambda$, this is as it should be. When $L_{0}=L_{0}^{g}$, given by the Sugawara formula (2.5), we know that its value on these states is $\lambda(\lambda+2 \varrho) /(x+\tilde{h}) \psi^{2}$ and so this must be the value of $-\delta$. Using this, the expression for $\Delta_{x, \lambda}(z, \theta)$ given by (A.7) simplifies to

$$
\Delta_{\lambda, x}(z, \theta)=\sum_{t \in \Lambda_{r}^{\nu}} z^{\left[(\lambda+\varrho+t)^{2}-\varrho^{2}\right] /(x+\tilde{h}) \psi^{2}} \sum_{\sigma \in W(g)} \varepsilon(\sigma) e^{i \sigma(\lambda+\varrho+t) \cdot \theta} .
$$

Formulae (A.6), (A.8), and (A.9) constitute the Weyl-Kac formula.

For $g=\operatorname{su}(2)$, the dual Coxeter number $\tilde{h}=2$ and it is customary to take $\psi=1$ so that $\varrho=\frac{1}{2}$. Then replacing $x$ by $N$ and $\lambda$ by $l$, we obtain Weyl-Kac formula in the form of Eq. (2.36). 
Acknowledgements. We are grateful to E. Corrigan, D. Fairlie, Z. Qiu, S. Shenker, and especially D. Friedan for interesting and useful conversations and communications. A.K. is grateful to the U.K. Science and Engineering Research Council for a Studentship.

\section{References}

1. Goddard, P., Kent, A., Olive, D.: Virasoro algebras and coset space models. Phys. Lett. 152 B, 88 (1985)

2. Polyakov, A.M.: Conformal symmetry of critical fluctuations. JETP Lett. 12, 381 (1970); Belavin, A.A., Polyakov, A.M., Zamolodchikov, A.B.: Infinite conformal symmetry in twodimensional quantum field theory. Nucl. Phys. B 241, 333 (1980)

3. Friedan, D., Qiu, Z., Shenker, S.: In: Vertex operators in mathematics and physics. Lepowsky, J. et al. (eds.). MSRI publications No. 3, Berlin, Heidelberg, New York: Springer 1984, p. 419; Conformal invariance unitarity and critical exponents in two dimensions. Phys. Rev. Lett.52, 1575 (1984)

4. Goddard, P., Olive, D.: Kac-Moody algebras, conformal symmetry and critical exponents. Nucl. Phys. B 257 [FS 14], 226 (1985)

5. Friedan, D., Qiu, Z., Shenker, S.: Superconformal invariance in two dimensions and the tricritical Ising model. Phys. Lett. 151 B, 37 (1985)

6. Ramond, P.: Dual theory for free fermions. Phys. Rev. D 3, 2415 (1971)

7. Neveu, A., Schwarz, J.H.: Factorizable dual model of pions. Nucl. Phys. B31, 86 (1971); Quark model of dual pions. Phys. Rev. D 4, 1109 (1971)

8. Sugawara, H.: A field theory of currents. Phys. Rev. 170, 1659 (1968)

Sommerfield, C.: Currents as dynamical variables. Phys. Rev. 176, 2019 (1968)

Coleman, S., Gross, D., Jackiw, R.: Fermion avatars of the Sugawara model. Phys. Rev. 180, 1359 (1969);

Bardakci, K., Halpern, M.: New dual quark models. Phys. Rev. D 3, 2493 (1971)

Dashen, R., Frishman, Y.: Four-fermion interactions and scale invariance. Phys. Rev. D 11, $2781(1975)$

9. Kac, V.G.: Infinite dimensional Lie algebras. Boston: Birkhäuser 1983

10. Goddard, P.: Kac-Moody algebras: representations and applications. DAMTP preprint 85/7

11. Olive, D.: Kac-Moody algebras: an introduction for physicists. Imperial College preprint $\mathrm{TP} / 84-85 / 14$

12. Rocha-Caridi, A.: In: Vertex operators in mathematics and physics. Lepowsky, J. et al. (eds.). MSRI publications No. 3, p. 451. Berlin, Heidelberg, New York: Springer 1984

13. Frenkel, I.B., Kac, V.G.: Basic representations of Lie algebras and dual resonance models. Invent. Math. 62, 23 (1980)

14. Segal, G.: Unitary representations of some infinite dimensional groups. Commun. Math. Phys. 80, 301 (1981)

15. Goddard, P., Olive, D.: In: Vertex operators in mathematics and physics. Lepowsky, J. et al. (eds.). MSRI publications No. 3, p. 51. Berlin, Heidelberg, New York: Springer 1984

16. Feigin, B.L., Fuchs, D.B.: Funct. Anal. Appl. 17, 241 (1983)

17. Kent, A.: Papers in preparation

18. Bardakci, K., Halpern, M.B.: New dual quark models. Phys. Rev. D3, 2493 (1971)

19. Goddard, P., Kent, A., Olive, D.: In preparation

20. Kac, V., Todorov, I.: Superconformal current algebras and their unitary representations. Commun. Math. Phys. 102, 337-347 (1985)

21. Altschuler, D.: University of Geneva preprint (1985) UGVA-DPT 1985/06-466

Communicated by A. Jaffe 
